\title{
Psychiatric out-patient clinics for older adults: highly regarded by users and carers, but irreplaceable?
}

\section{AIMS AND METHOD}

The aim of this study was to explore older users' and carers' views of attending out-patient clinics for older adults. A questionnaire was designed to be distributed to all patients attending out-patient clinics for a period of 1 month in January 2007.

\author{
RESULTS \\ The response rate for returning the \\ completed questionnaire was $71 \%$, \\ and $95 \%$ of these respondents were \\ satisfied with their overall experi- \\ ence of attending out-patient clinics. \\ Although satisfaction levels were \\ high with every aspect of out-patient \\ clinics, $49 \%$ preferred the hypoth- \\ etical option of being seen at home.
}

\author{
CLINICAL IMPLICATIONS \\ The role of psychiatric out-patient \\ departments for older adults merits \\ further research, comparing cost and \\ user/carer acceptability with home \\ or general practitioner surgery- \\ based treatment.
}

Current government strategy in England is to reduce out-patient attendance, replacing it with people being seen in primary care (Rowland et al, 2006). There is little evidence to support this strategy, in terms of quality of patient care or patient choice.

Psychiatric out-patient attendance for working-age adults is declining, as a result of the rise of crisis assessment and home treatment teams, and the fact that missed appointments are common in working-age adults (Rusius, 1995; Goode, 1997). By contrast, psychiatric out-patient attendances have increased for older adults since 2001, with National Institute for Health and Clinical Excellence (NICE; Clegg et al, 2000) guidance on cognitive enhancers stipulating that antidementia drugs should be initiated and monitored by secondary care. Memory clinics, memory management services and non-medical prescribing have developed piecemeal (Lindesay et al, 2002), although in many areas cognitive enhancers are prescribed and monitored in traditional psychiatric out-patient departments where people with chronic functional illness are also monitored and treated.

A previous audit in Sheffield found that of 78 older people attending psychiatric out-patient clinics satisfaction levels of the service were high and attendance was in excess of $85 \%$ (K. Newton \& C. Rusius, personal communication, 2006). This current questionnaire-based study sought to extend this audit, ask older users' and carers' their views of attending psychiatric out-patient clinics and seek their views about possible alternative provision.

\section{Method}

A questionnaire was derived comprising 28 questions requiring a tick box Likert scale answer (see online supplement). Some of the questions were taken from a previous internal audit of patient's satisfaction conducted by Sheffield Care Trust that included positive and negative statements, with additional questions from similar questionnaire studies in other populations. The questionnaire contained questions on demographics, convenience and general experience of psychiatric outpatient clinics (out-patients), with space for additional qualitative comments. After being piloted for acceptability, the questionnaire was distributed to all patients attending two psychiatric out-patient departments for older people during the month of January 2007. Both departments are stand-alone buildings in Sheffield hospitals (Northern General and Nether Edge), serving both general adult and old age psychiatry, with dedicated receptionists and part-time support worker time. Most clinics contained a mixture of 'organic' and 'functional' patients, although one weekly memory clinic compromised only people attending with a diagnosis of likely early dementia. Consultants, specialist registrars, staff grades and senior house officers conducted the clinics under supervision.

The questionnaires were given to patients by receptionists on arrival and were collected by the support worker, the receptionist or the psychiatrists performing the clinic. Most people completed them while they sat in the waiting room prior to being seen. New patients attending for the first time were given the option of returning the questionnaire by post, although most completed them before leaving the department. Patients, carers (or both patients and carers together) completed the anonymised questionnaires. For individuals with advanced dementia the carer alone completed the questionnaire.

\section{Results}

Computerised data showed that there were 336 older adult out-patient attendees during the study period, of whom approximately 240 were invited to complete questionnaires. Not every attendee was offered the questionnaire as clinic staff were busy or the authors were not in out-patients that day. Only one person refused to complete the questionnaire, but an additional 69 were excluded, as they had not been fully completed. 


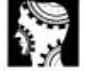

original papers
A total of 171 fully completed questionnaires were included. Of these, $37 \%$ were completed by the patient alone and $63 \%$ with the assistance of a carer/relative. In total, $8 \%$ had driven themselves or walked to the clinic, $52 \%$ had been brought by a carer/relative in their car, $32 \%$ by taxi. Only $15 \%$ identified that travelling to outpatients was difficult to arrange.

Seventy per cent identified their reason for attending as 'memory problem', although a formal diagnostic breakdown was not performed. In total, $14 \%$ were new/first-time attendees, 86\% were follow-up appointments.

Overall, patients and carers were highly satisfied with every aspect of attending out-patients (Table 1) replicating the finding of the previous audit. Satisfaction levels were equally high for both clinics; $95 \%$ agreed that they were overall satisfied with attending out-patients.

The majority of qualitative comments were highly appreciative of the services, for example:

- 'I have always found the staff most helpful, and it is good to talk to the doctor who always listens to all the problems which we come across.'

- 'I am highly pleased with the treatment.'

- 'The clinic has benefited my grandmother immensely. At times she wouldn't leave her home, she will always attend the clinic. The staff are excellent.'

- 'I would appreciate it if my mother who is in a nursing home can be seen there, as she gets very agitated when taken out of her usual environment.'

In terms of potential reorganisation, $83 \%$ of respondents agreed/strongly agreed that out-patients was different from seeing their general practitioner (GP)

Table 1. Percentage of users/cares rating 'agree' or 'strongly agree' on five-point scale Users/carers rating 'agree' or 'strongly agree', \%

The receptionist staff are pleasant

I am treated with respect

Professional/doctor listens to me

It is easy to get in and out of the building

The professional is trustworthy

My information is treated confidentially

I do not feel intimidated

The appointment times are convenient

Professional/doctor accepts my opinion

The reasons for giving medication are

explained

Psychiatric out-patients is useful to me

Psychiatric out-patients is a better service

than seeing a general practitioner for mental

health difficulties

My mental health has not benefited from

attending psychiatric out-patients

My appointment times are convenient

There is enough flexibility to change

appointments

I spend too long in the waiting room (i.e. seeing their GP could not replace out-patients). Overall, 75\% felt out-patients was a better service for mental health difficulties than seeing their GP - only $6 \%$ did not. When asked the question: 'If we were to re-organise our services, which of these would you prefer?', users/carers responded as follows:

- home visit from mental health worker (not necessarily a doctor): $49 \%$;

- continue to attend out-patients: $42 \%$;

- don't know: 9\%.

\section{Discussion}

The only other published study examining psychiatric out-patient clinics for older adults explored replacing out-patients with community clinics, i.e. seeing people at home (Benbow, 1990). This model has not been adopted, either in rural or urban catchments areas. This is the first published study assessing user and carer views about attending psychiatric out-patients for older people.

The main finding was that satisfaction with all aspects of the service was high, both for organisation of the clinics and the interaction with the psychiatrists of all grades; reflected also by the high attendance rate at outpatients. The two out-patient departments surveyed in Sheffield are not unusual in their service provision factors, although it is not clear if the results are generalisable.

This questionnaire study, although simple, has some strength that increases its clinical relevance. The study sample consisted of a cross-section of people with common mental illnesses of old age including depressive disorder, anxiety disorder and dementia. Most people were attending for follow-up appointments (86\%), hence it was easy for them to complete the questionnaire based on their previous experiences. New patients were requested to complete the questionnaire after they had been seen and were given the option to post it back if they found it more convenient. The response rate of completing the questionnaire was high as most patients and carers found it simple and easy to understand. Only a few individuals, particularly those with cognitive impairment, found the questionnaire lengthy and time consuming.

There are obvious limitations of this type of study. Surveys rely on a self-report method of data collection, so positive response bias, intentional deception, poor memory or misunderstanding of questions can all contribute to inaccuracies in the data, which may not necessarily be transferable to other out-patient settings. The study did not explore the views of service users in depth and therefore many questions remain unanswered.

Owing to resource limitations, the waiting times were not recorded; with hindsight this could have strengthened the overall findings of the study, as satisfaction with services may be linked to time kept waiting. However, in this study $86 \%$ disagreed or strongly disagreed that they had spent too long in the waiting area.

As a result of the large response rate that the questionnaire generated it is easy to contemplate that most service users found it easy to complete; however, 
69 questionnaires were excluded due to incomplete response. The incomplete response may be linked to the difficulties some of the people may have experienced while completing the questionnaire. These difficulties could be related to the framing of questions as well as the length of questionnaire, as reported by a few patients with cognitive impairment. However, with hindsight, recording apparent reasons for not completing the questionnaire would have been quite useful to explain fully the incomplete response. Furthermore, this information could have been used for future similar questionnaire-based studies. It is also, therefore, a possibility that if these incomplete questionnaires or the reasons for not completing them were included, it may have affected the overall satisfaction levels.

The key finding of the study was that, despite high satisfaction levels with out-patients, nearly half of users thought it preferable to be visited at home by another mental health professional (not necessarily a doctor). Although the questionnaire did not seek reasons for this statement, we feel that convenience factors for users and carers were the main reasons that people would prefer to be seen in their own home. It could be concluded that non-medical prescribing could be developed further, particularly for people taking cognitive enhancers. However, if services were to be reconfigured, staff training/supervision, staff time and transport costs would be factors to be considered, alongside patient choice; individuals attending out-patients in Sheffield have access to facilities for physical examination, phlebotomy and a specialist pharmacy on site, which would not be available in peoples' own homes.

There are thus many areas for further research including assessment of the practicalities and acceptability of home-based community clinics, and a cost-benefit analysis of different models of service provision. As out-patient clinics are highly acceptable to service users (at least in Sheffield) and NICE guidance for cognitive enhancers requires frequent assessments, out-patient clinics for older adults should not be closed on the basis of changes in general adult psychiatry until alternatives have been evaluated.

\section{Declaration of interest}

None.

\section{References}

BENBOW, S. M. (1990) The community clinic - its advantages and disadvantages. International Journal Geriatric Psychiatry, 5, 119-121. clinics in the British Isles. International Journal Geriatric Psychiatry, 17, $41-47$.

CLEGG, A., BRYANT, J., NICHOLSON,T. et al (2000) Clinical and Cost Effectiveness of Donepezil,

Rivastigmine and Galantamine for Alzheimer's Disease. National Institute for Health and Clinical Excellence.

GOODE, L. (1997) Null contacts by clients referred by general practitioners and hospital consultants. Clinical Psychology Forum, 99, 32-35.

LINDESAY, J., MARANELKAR, M., VAN DIEPEN, E. \& WILCOCK, G. (2002) The

ROWLAND, M., MCDONALD, R. \& SIBBALD, B. (2006) Outpatient Services and Primary Care: A Scoping Review of Research into Strategies for Improving Outpatient Effectiveness and

Efficiency. National Institute for Health Research (http://www. sdo.Ishtm.ac.uk)

RUSIUS, C. W. (1995) Improving outpatient attendance using postal appointment reminders. Psychiatric Bulletin, 19, 291-292.

*Rashi Negi Specialist Registrar in Old Age Psychiatry, Michael Carlisle Centre, Nether Edge Hospital Osborne Road, Sheffield S11 9BF, email: rashinegi@hotmail. com, Jeremy Seymour Consultant in Old Age Psychiatry, Nether Edge Hospital, Sheffield, Christopher Flemons Senior House Officer in Psychiatry, Longley Centre, Sheffield, Matthew Impey Senior House Officer in Psychiatry, Longley Centre, Sheffield, NicolaThomas Senior House Officer in Psychiatry, Longley Centre, Sheffield, Rachael Witrylak Senior House Officer in Psychiatry, Nether Edge Hospital, Sheffield

\section{REENA KHIROYA, TIM WEAVER AND TONY MADEN}

\section{Use and perceived utility of structured violence risk assessments in English medium secure forensic units}

\section{AIMS AND METHOD}

We surveyed the usage and perceived utility of standardised risk measures in 29 forensic medium secure units (a $62 \%$ response rate).

\section{RESULTS}

The most common instruments were Historical Clinical Risk-20 (HCR-20) and Psychopathy Checklist - revised
$(P C L-R)$; both were rated highly for utility. The Risk Matrix 2000 (RM2000), Sex Offender Risk Appraisal Guide (SORAG) and Static- 99 were the most common sex offender assessments, but the Sexual Violence Risks-20 (SVR-20) was rated more positively for its use of dynamic factors and relevance to treatment.

\author{
CLINICAL IMPLICATIONS \\ Most medium secure units use struc- \\ tured risk assessments and staff view \\ them positively. As HCR-20 and \\ PCL-R/PCL-SV (Psychopathy \\ Checklist - Screening Version) are \\ so widely used they should be the \\ first choices considered by other \\ services.
}

Violence risk assessment is central to the work of forensic mental health services. Standardised methods of assessment have become more common but there is great variation between services in the use of such instruments.
The alternatives to clinical assessment alone are actuarial methods (e.g. using the Violence Risk Appraisal Guide (VRAG); Quinsey et al, 1998) that prescribe the collection and interpretation of data relevant to risk 